\title{
PAPER
}

\section{Sexual function in men with cauda equina lesions: a clinical and electromyographic study}

\section{S Podnar, C Oblak, D B Vodušek}

See end of article for authors' affiliations .....................

Correspondence to: Dr S Podnar, Institute of Clinical Neurophysiology, Division of Neurology, University Medical Centre Liubliana, SI-1525 Ljubliana, Slovenia; simon.podnar@kcli.si

Received 2 May 2002 In revised form

16 July 2002

Accepted

5 September 2002

\begin{abstract}
Objective: To investigate the effects of cauda equina lesions on sexual function in men.
Methods: Sexual function was investigated in 46 men with long standing cauda equina/conus medullaris lesions. All had clinical and radiological findings supporting the diagnosis. The validated Slovene translation of the international index of erectile function (IIEF) was used. The responses were scored and sexual dysfunction categorised as absent, mild, moderate, or severe. The number of patients receiving help for sexual dysfunction was noted. Neurological examination of the trunk and lower limbs, electromyographic (EMG) evaluation of the sacral reflex, and quantitative EMG of the external anal sphincter muscles were done.

Results: Severe sexual dysfunction was reported by $35 \%$ of patients, moderate dysfunction by $24 \%$, and slight dysfunction by $26 \%$; normal sexual function was reported by $15 \%$. Orgasmic function was slightly more impaired than erectile function, and sexual desire slightly less. The patients' age, but no findings on clinical neurological or EMG examination, correlated with sexual function. Only five men had received medical attention for sexual dysfunction.

Conclusions: There is significant sexual impairment in men with lesions of the cauda equina or conus medullaris. This is poorly correlated with neurological and EMG findings and has received insufficient medical attention.
\end{abstract}

$\mathrm{T}$ he spinal cord tapers to its end, the conus medullaris, usually at the lower edge of the first lumbar vertebra in adults. From the conus medullaris, lumbar and sacral nerve roots arise, forming a nerve bundle within the spinal canal called the cauda equina. Spinal roots leave the cauda equina consecutively at their appropriate neural foramina.

Injuries to the cauda equina are mostly caused by compression from herniated intervertebral discs, fractured spinal fragments, tumours, haematomas, or abscesses. In the case of trauma (which mainly affects the lower thoracic and the upper lumbar vertebrae) the conus medullaris or the cauda equina, or both, may be affected, and it is difficult to separate the two conditions. In the present study we used the term "cauda equina lesion," disregarding the exact location of the lesion. Other much rarer conditions (ischaemic insults, inflammatory, infectious, and toxic conditions) may also be responsible for cauda equina damage. In spite of the serious consequences of such lesions on urinary, anorectal, and sexual function, and their potential to cause chronic pain syndromes, data obtained in systematically studied populations of these patients are still inadequate. ${ }^{1-4}$ The exact incidence and prevalence of lesions to the cauda equina in a general population are not known, but it is estimated that they present $1-5 \%$ of spinal pathology. ${ }^{35}$

In the present study, we report the sexual function of men with long standing lesions of the sacral nerve roots, and correlate it with findings on clinical neurological examination and quantitative electromyography (EMG) in the external anal sphincter muscles. In addition to clinical examination, EMG of the external anal sphincter is considered a useful optional investigation for defining lesions better within the lower sacral reflex arc. In patients with lesions to the cauda equina, no quantitative EMG study has yet been published.

\section{METHODS}

A group of 46 men was recruited either from the database of the Institute of Clinical Neurophysiology in Ljubljana (34 patients), or from the database of the Institute of Republic of Slovenia for Rehabilitation ( 12 patients). In addition to a history suggesting the cause, all patients had some perianal sensory loss or definite pathological denervation activity on EMG examination performed early (3-10 weeks) after the onset of the condition; all patients also had radiological findings supporting a cauda equina injury. The national ethics committee of Slovenia approved the study, and all subjects provided informed consent.

At the clinical and EMG examination, all patients were asked about erectile and ejaculatory dysfunction. Afterwards, the validated Slovene translation of the international index of erectile function (IIEF) ${ }^{6}$ was sent to all patients. The responses to 15 questions of the IIEF were scored using five or six point scales; better sexual function scored higher. The first 10 questions (Q1-10) were scored 0-5, and the last five questions (Q11-15) scored 1-5. Six questions (Q1-5, Q15) concerned erectile function, three (Q6-8) concerned intercourse satisfaction, two (Q9-10) concerned orgasmic function, two (Q11-12) concerned sexual desire, and two (Q13-14) concerned overall satisfaction (table 1, figs 1 and 2). ${ }^{6}$ Each area of sexual activity was first scored separately, and then a general score of sexual function was obtained by summation of the individual scores. Sexual dysfunction in the individual domains and the general score were categorised as absent, or as mild, moderate, or severe sexual dysfunction (table 1 ).

Neurological examination of the trunk and lower limbs was undertaken in all patients. This included: power of knee flexion (graded as normal, slightly reduced, moderately reduced, or severely reduced/no limb movement), briskness of ankle jerks (graded as normal, diminished, or absent), and perianal sensation (graded separately on each side as normal, mildly reduced, moderately reduced, or severely reduced/absent).

Abbreviations: IIEF, international index of erectile function; MUP, motor unit potentials 
Table 1 Sexual dysfunction in patients with chronic lesions of the cauda equina: a group of 36 patients was assessed using the international index of erectile function (IIEF) questionnaire

\begin{tabular}{|c|c|c|c|c|c|c|c|c|}
\hline \multirow[b]{3}{*}{ Function } & \multicolumn{8}{|c|}{ Dysfunction } \\
\hline & \multicolumn{2}{|l|}{ Absent } & \multicolumn{2}{|l|}{ Mild } & \multicolumn{2}{|c|}{ Moderate } & \multicolumn{2}{|c|}{ Severe } \\
\hline & Score & $\%$ & Score & $\%$ & Score & $\%$ & Score & $\%$ \\
\hline Erectile & $26-30$ & 22 & $17-25$ & 25 & $11-16$ & 11 & $1-10$ & 41 \\
\hline Orgasmic & $9-10$ & 17 & $7-8$ & 11 & $3-6$ & 25 & $0-2$ & 47 \\
\hline Sexual desire & $9-10$ & 14 & $7-8$ & 28 & $5-6$ & 19 & $2-4$ & 39 \\
\hline Intercourse satisfaction & $11-15$ & 17 & $8-10$ & 28 & $4-7$ & 19 & $0-3$ & 36 \\
\hline Overall satisfaction & $9-10$ & 6 & $7-8$ & 22 & $5-6$ & 36 & $2-4$ & 36 \\
\hline General index & $60-75$ & 17 & $42-59$ & 25 & $22-41$ & 22 & $0-21$ & 36 \\
\hline
\end{tabular}

Sexual dysfunction ranges for IIEF scores and percentages of patients with each level of dysfunction are shown.

EMG of the external anal sphincter muscle was undertaken using a standard concentric EMG needle (37 mm long; No 22583, Oxford Medical, Old Woking, Surrey, UK), and an advanced EMG system (Keypoint, Medtronic Functional Diagnostics, Skovlunde, Denmark) with standard settings (filters: $5 \mathrm{~Hz}$ to $10 \mathrm{kHz}$ ). A precisely standardised examination protocol was followed. ${ }^{7}$ At each site, the number of continuously firing motor unit potentials (MUP) was first counted using multi-MUP analysis. MUP counts obtained in patients during relaxation were compared with normative data. ${ }^{8}$ With a needle electrode inserted into the subcutaneous external anal sphincter muscle, the increase in MUP firing to perianal touch (that is, sacral reflex activation) was noted (graded as normal, mildly reduced, moderately reduced, or
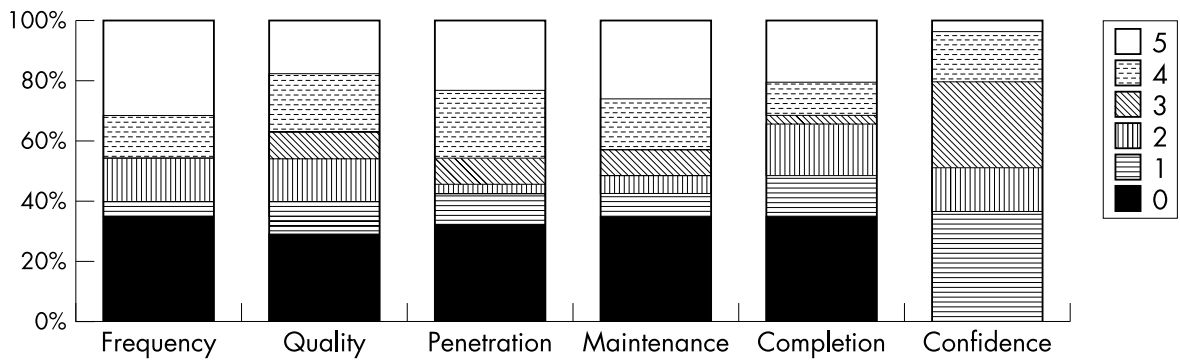

Figure 1 Erectile function in 36 men with chronic lesion of the cauda equina. Using the international index of erectile function (IIEF) questionnaire, ${ }^{6}$ responses on frequency of obtaining an erection (question 1, Q1), having an erection hard enough for penetration (Q2), ability to penetrate the partner (Q3), ability to maintain erection after penetration (Q4), and difficulty in maintaining erection until completion of intercourse (Q5), together with confidence to achieve and maintain an erection (Q15) are presented. Q1-5 were scored 0-5 (0, no sexual activity; 1, almost never/never; 2, rarely; 3, sometimes; 4, usually; 5, almost always/always). Q15 was scored 1-5 (1, very low; 2, low; 3, moderate; 4, high; 5, very high). In IIEF all questions evaluate sexual activity in the previous (last) four weeks. Better sexual function scores higher. Note the slight decrease in scores on successive questions $(Q 1-5)$, and a very low confidence in achieving and maintaining erection during sexual intercourse (Q15).

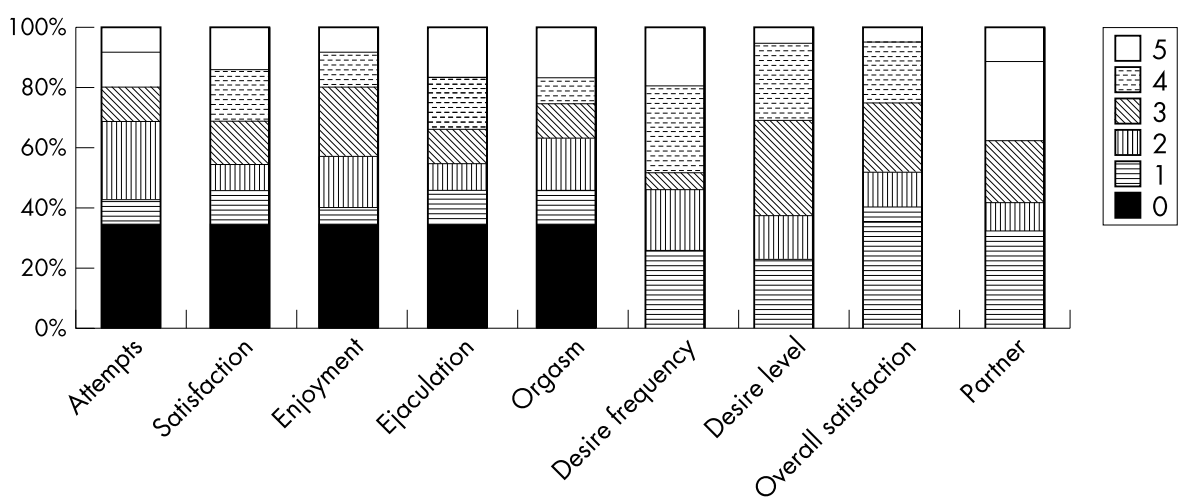

Figure 2 Responses of 36 men with chronic cauda equina lesion to the international index of erectile function (IIEF) questionnaire. ${ }^{6}$ In IIEF all questions evaluate sexual activity in the previous (last) four weeks. Frequency of attempts to have sexual intercourse (Q6), satisfaction (Q7), and enjoyment (Q8) with sexual intercourse evaluate intercourse satisfaction. Frequency of ejaculation (Q9) and frequency of feeling of orgasm (Q10) evaluate ejaculatory function. Frequency (Q1 1) and level (Q12) of sexual desire evaluate that domain of sexual life. Overall satisfaction with sexual life (Q13) and satisfaction with sexual relationship with partner (Q14) evaluate overall sexual satisfaction. ${ }^{6}$ Note that Q6-10 were scored 0-5 and Q1 1-14 were scored 1-5. Better sexual function scored higher. In Q7, Q9, Q10, and Q1 1, the scale was the same as in Q1-5, and in Q12 the scale was the same as in Q15 (fig 1). Q6 was scored as follows: 0, no attempts; 1, one to two attempts; 2, three to four attempts; 3, five or six attempts; 4, seven to 10 attempts; 5, 11 or more attempts; Q8 was scored as follows: 0, no intercourse; 1, no enjoyment; 2, not very enjoyable; 3 , fairly enjoyable; 4 , highly enjoyable; 5, very highly enjoyable; Q13 and Q14 were scored as follows: 1 , very dissatisfied; 2, moderately dissatisfied; 3, about equally satisfied and dissatisfied; 4, moderately satisfied; 5 , very satisfied. 
severely reduced/absent). ${ }^{7}$ During these procedures, and when necessary by appropriate voluntary activation, MUPs were sampled using multi-MUP analysis. MUP indices of area, duration, and number of turns ${ }^{9}$ were evaluated. Mean values and "outlier" limits of individual MUPs were compared with appropriate normative data. ${ }^{10}$ EMG abnormalities were graded (in decreasing severity) as follows:

(1) complete atrophy of both the subcutaneous and the deeper external anal sphincter muscles bilaterally;

(2) incomplete external anal sphincter reinnervation (complete atrophy, or values of MUP indices below the normative range in at least one external anal sphincter muscle);

(3) MUP indices above the normative range in more than half the muscles examined;

(4) MUP indices above the normative range in less than half the muscles examined;

(5) no abnormalities on MUP analysis.

Similarly, general scores of clinical findings from both sides were also determined.

The number of men evaluated for their sexual dysfunction by urologists or andrologists, treatments recommended, and the effect of treatments were noted.

\section{Statistics}

Correlations between the total sexual function score and the patients' age, duration of neural damage, clinical findings (power of knee flexion, perianal sensation, ankle jerks), and EMG findings (MUP count, sacral reflex activation, MUP indices) were assessed by the non-parametric Spearman correlation coefficient. We also analysed the effect of age, aetiology, duration, and level of the neural lesion, perianal sensation, and the grade of EMG abnormality on different domains of sexual function (erectile function, intercourse satisfaction, orgasmic function, sexual desire and overall satisfaction) and on the general score of male sexual function, using multiple linear regression analysis by MATLAB (The MathWorks Inc, Natick, Massachusetts, USA).

\section{RESULTS}

The median age of our patients was 47 years (range 21 to 78 ), and the median time since the lesion to the cauda equina was 22 months (range 5 to 240). In 20 men, the lesion was caused by intervertebral disc herniation, in 16 by spinal fracture, in four either by surgery to the intervertebral disc (3) or to the abdominal aorta (1), in three by a spinal tumour, and in a further three by a spinal haematoma in the lumbar region, a spinal abscess, and chronic arachnoiditis, respectively.

The completed IIEF was received from 36 patients (table 1 , figs 1 and 2). More than half the patients reported moderate or severe sexual dysfunction. Different areas of the men's sexual function were similarly affected, with orgasmic dysfunction (Q9-10) reported to be slightly more and sexual desire (Q1112) slightly less affected than erectile function (Q1-5, Q15). Erectile function scores progressively diminished with consecutive IIEF questions. Thus, although 12 patients $(30 \%)$ were (almost) always able to achieve an erection during sexual activity (Q1), only one (3\%) was very highly confident of achieving and maintaining an erection (Q15). The most affected domain was intercourse satisfaction, with only one patient highly enjoying it (Q8). More than half the patients were dissatisfied and only a quarter were satisfied with their sexual life (Q13). These data, obtained by a self administered IIEF questionnaire, were in accordance with data obtained by a structured interview.

In the remaining 10 patients, who did not return the IIEF questionnaire, one had normal, three mild, three moderate, and three severe erectile and ejaculatory impairment (data collected by structured interview). Thus of the 46 patients, 16
(35\%) had severe, 11 (24\%) moderate, and $12(26 \%)$ slight sexual dysfunction. The remaining seven men (15\%) claimed to have normal sexual function. Although this was not directly asked for, seven men (44\%) with severe sexual dysfunction and without the ability to achieve ejaculation during sexual intercourse reported spontaneous ejaculations of semen during sleep.

One patient was totally and another partially wheelchair bound. The remaining patients were ambulant, some with varying degrees of lower limb paresis.

On neurological examination, knee flexion power was normal in 22 patients, slightly reduced in 20, moderately reduced in three, and severely reduced in one. Ankle jerks were normal in eight patients, reduced in 23, and absent in 15. Almost all men (43) had a clear perianal sensory deficit at least unilaterally. When the left and right sides were graded individually in patients with sensory loss, perianal sensation was normal on six sides, mildly/moderately reduced on 24 sides, severely reduced on 33 sides, and absent on 23 sides. When assessment of both sides was combined into one grade, normal sensation was present in three patients, mild reduction in 11 , moderate reduction in nine, severe reduction in 15 , and the remaining eight patients did not feel any perianal touch or pinprick.

On EMG examination, the subcutaneous and deeper external anal sphincter muscles were bilaterally completely atrophied in four of 46 patients, incompletely reinnervated in nine, severely neuropathic in 22 , slightly to moderately neuropathic in six, and normal in five. The MUP count during relaxation was below the normal range in at least one external anal sphincter muscle in 21 of 45 patients (47\%).

Sacral reflex activation was normal in seven patients, mildly reduced in nine, moderately reduced in nine, severely reduced in eight, and absent in 13. Of 16 patients with normal or mildly reduced sacral reflex activation, only two older than 60 years had severe sexual dysfunction, and of 21 men with severely reduced or absent reflex activation, eight had severe sexual dysfunction.

Age was the only factor that was significantly correlated with overall sexual function as assessed by the nonparametric Spearman correlation coefficient, and on multiple linear regression analysis it also importantly affected all five domains of male sexual function (erectile function, intercourse satisfaction, orgasmic function, sexual desire, and overall satisfaction), as well as the general score of male sexual function $(p<0.0001)$. In men younger/older than 47 years (the median age in our population), sexual function was severely impaired in $17 \% / 54 \%$, moderately impaired in $29 \% / 18 \%$, mildly impaired in $29 \% / 23 \%$, and normal in $25 \% / 5 \%$ (mean IIEF scores, 46/22).

None of the other variables evaluated using these statistical techniques (aetiology, duration and level of neural damage, power of knee flexion, perianal sensation, ankle jerks, MUP count, sacral reflex activation, MUP indices) had any effect on sexual function of our patients $(p>0.05)$.

Only four patients were evaluated by a urologist for sexual dysfunction. Two were offered oral sildenafil citrate (Viagra; Pfizer, New York, USA), one intracavernosal injections, and one a vacuum device. All these approaches improved erection but did not increase pleasure during intercourse. One of our patients (totally wheelchair bound) was seen by an andrologist, who took a sperm sample and recommended the use of a vibrator, which, however, did not enable him to achieve ejaculation.

\section{DISCUSSION}

The cauda equina conveys sensory fibres from the sacral dermatomes, motor nerve fibres innervating lower sacral myotome skeletal muscles, and sacral parasympathetic fibres. ${ }^{11}$ Thus in patients with cauda equina lesions the finding of pronounced sexual dysfunction, perineal sensory deficit, and EMG abnormalities is not unexpected. ${ }^{11}$ 
Cell bodies of motor and sacral parasympathetic neurones reside within the Onuf and intermediolateral nuclei of the conus medullaris, respectively, and these also receive input from afferent sacral nerve fibres. The effects of lesions to the cauda equina and the conus medullaris are, as a result, similar and it is difficult to distinguish between them clinically. In particular, on multiple linear regression analysis our patients with spinal fractures-most of whom had Ll fracturesprobably had at least some involvement of the conus medullaris (with or without root damage). We consider conus medullaris lesions improbable in patients with disc herniations, which in all our cases occurred below the L3 level. Cauda equina lesions are often asymmetrical, whereas conus medullaris lesions are not. In the present study, we did not try to distinguish between these two possible locations of neural injury to the lower sacral segments.

Parasympathetic fibres innervate the penile vascular structures and are considered a proerectile pathway. ${ }^{12}$ In addition, contraction of the ischiocavernosal and bulbospongiosal striated muscles, located at the penile crus and innervated by the S2-S4 somatic motor fibres, plays a contributory role in penile erection. ${ }^{13}$

Sensory fibres from the lower sacral dermatomes convey genital tactile sensation, which is important for conscious appreciation of genital stimulation and for reflexogenic erections. Accordingly, spinal coordination of the various autonomic and somatic nuclei is required for a rigid and sustained reflex penile erection. ${ }^{13}$

In contrast, sympathetic innervation of the genital organs bypasses the cauda equina and is thus not affected by damage to it. The sympathetic outflow leaves the spinal cord at the thoracolumbar (T10-L2) segments, travels in the hypogastric nerves, and reaches the genital structures by way of the pelvic plexuses. It is likely to be responsible for the basal antierectile tone present during the flaccid state of the penis, ${ }^{12}$ but it also promotes psychogenic erections. ${ }^{14}$

The main role of the sympathetic innervation is emission of semen from the vas deferens and seminal vesicles into the posterior urethra, and closure of the bladder neck, thus preventing retrograde ejaculation. Ejaculation is then reinforced by rhythmic contractions of the ischiocavernosal and the bulbospongiosal muscles. A spinal cord centre for ejaculation has been proposed at the T12/Ll level. ${ }^{15}$ It is thought that it receives facilitatory influences from the brain and from the genitalia through sensory fibres. Almost half our men with severe sexual dysfunction reported spontaneous ejaculation of semen during sleep, which supports sparing of the sympathetic innervation in this population.

Our patients reported slightly more pronounced impairment of ejaculatory (orgasmic) function than of erectile function. To achieve ejaculation during sexual activity, the spinal centre for ejaculation needs to be activated. This depends on sensory stimulation from the genitalia (or central activation). In addition, ejaculation of semen from the urethra depends on rhythmic contraction of the periurethral striated musculature. Thus, although efferent ejaculatory (sympathetic) fibres are preserved in lesions of the cauda equina, both the sensory fibres from the anogenital region and the motor innervation of the perineal musculature are affected.

Sexual desire, which was least affected in our study, is a function of the brain. In our patients it could be impaired by previous negative sexual experiences caused by erectile dysfunction.

To our knowledge, this study is the first to evaluate sexual function systematically in a larger population of men with cauda equina lesions. It revealed significant sexual impairment in this population, with more than a third of the patients having severe sexual dysfunction, a quarter each having moderate or mild dysfunction, and only 15\% having (self reported) normal function. None of the relevant previous studies used IIEF. Thus a comparison of our results with published data is difficult. However, it is evident that our patients had much worse sexual function than men of a similar age in the general population. At the age of 45 years (the median age of our population was 47 years), approximately $6 \%$ of men had complete erectile dysfunction, 19\% had moderate dysfunction, $17 \%$ had minimal dysfunction, and 55\% had no dysfunction. ${ }^{16}$ Furthermore, most of our patients related the onset of erectile dysfunction to the neural lesion.

Previous studies of patients with lesions to the cauda equina only briefly mentioned sexual function in small populations of men with lesions following surgery for prolapsed intervertebral discs. ${ }^{1-5}$ They reported complete loss of erectile function in one of nine men, ${ }^{5}$ two of six, ${ }^{3}{ }^{17}$ two of $10,{ }^{2}$ and in none of 12 , all of whom were sexually active before injury. ${ }^{4}$ In a further four of nine men, ${ }^{5}$ two of six, ${ }^{3}{ }^{17}$ and one of $12,{ }^{4}$ erectile function was impaired. In the study by Hellstrom et al, four of 10 men transiently lost erectile ability for several years, after which it normalised. ${ }^{2}$ In the study by Larsen and Hejgaard, five of 11 patients with cauda equina lesions had sexual dysfunction but their sex was not stated. ${ }^{1}$ Again, comparison of our results with these studies is difficult, but in general they reported better outcomes for sexual function after lesions to the cauda equina than in our patients.

There may be several reasons for this. All previous studies were done in populations of patients operated on for herniated intervertebral discs in large neurosurgical or orthopaedic centres, ${ }^{1-4}$ which contrasts with our population of patients with different aetiologies, recruited from the databases of a diagnostic and a rehabilitation centre. Patients from large neurosurgical or orthopaedic centres probably have better outcomes than the general population because they are likely to have had early decompression surgery. In contrast, rehabilitation centres treat patients with severe residual dysfunction. Furthermore, in our study patients were invited to participate by letter, and it is possible that this preferentially attracted patients with greater dysfunction. The IIEF used here may also be more sensitive than the clinical evaluations performed in published studies. ${ }^{1-4}$

Comparing our results with two other neurological disorders that affect young men-multiple sclerosis and spinal cord injuries-reveals a similar prevalence of sexual dysfunction, which affects about $70 \%$ of men with multiple sclerosis ${ }^{18}$ and $25-95 \%$ of men with spinal injuries. ${ }^{19}$ The main difference, however, between the population of patients with cauda equina lesions and these other two conditions is in the importance ascribed to sexual dysfunction. Only two of our patients were not ambulant, which contrasts to the situation in multiple sclerosis and spinal cord injuries. As a result, sexual dysfunction formed a larger proportion of their total disability. As the total distress that the patients experienced as a result of sexual dysfunction is not investigated by IIEF, it was not formally recorded in our patients. However, on the basis of the patients' histories, it seemed that young patients in particular were seriously distressed by their sexual disability.

In our study, age was a strong predictor of the severity of sexual dysfunction, with older patients reporting greater sexual deficits. This is in concordance with findings in the normal population. ${ }^{16}$ Part of this difference may reflect bias in reporting deficits. Although it has been established that self reporting techniques allow patient derived assessments outside the clinic and that they are appropriate for evaluating sexual function, ${ }^{20}$ it was our impression that older patients tended to overestimate their impairment (perhaps because of depression and the lack of a sexual partner). In contrast, it seems possible that some younger patients may have underestimated their sexual deficit. A 26 year old patient, five years after spinal fracture and with complete sacral anaesthesia/ analgesia, complete external anal sphincter muscle atrophy, and severe faecal and urinary incontinence, claimed to have normal erections, with only premature ejaculations. We have 


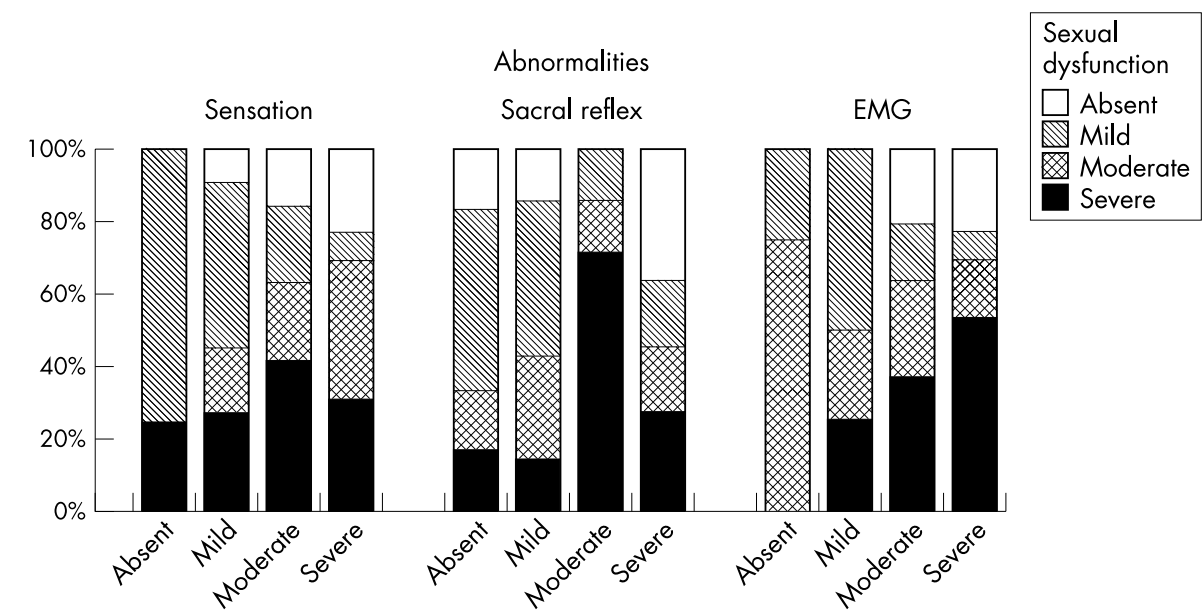

Figure 3 Correlation between perianal sensation, sacral reflex response, and motor unit potential (MUP) analysis on quantitative electromyography (EMG) of the external anal sphincter (EAS) muscle in 46 men with chronic lesions of the cauda equina. Note the poor correlation of clinical and EMG abnormalities with sexual (dys)function.

no partner report, nor could we perform a laboratory observation of his erection. A similar patient was described previously. ${ }^{2}$ Denial might be important in some patients, and therefore responses obtained by self administered sexual questionnaires need to be accepted with caution.

The aetiology of lesions was not important for the resulting sexual deficit, as patients with neural damage resulting from intervertebral disc herniation, spinal fractures, and other lesions had a similar severity of sexual dysfunction. However, it seemed that patients with iatrogenic lesions (disc and abdominal aneurysm surgery) and spinal haematomas had somewhat worse outcomes in terms of sexual function. In contrast, three patients with compression to the cauda equina by spinal tumours had the best preserved sexual function. Different pathophysiological mechanisms of injury and different degrees of compression probably caused these differences.

On neurological examination, knee flexion muscle power and briskness of the ankle jerks were not correlated with sexual dysfunction. This is not surprising as these evaluate mainly the S I segment, which may or may not be damaged in a neural lesion involving the cauda equina. S2 and S3 are considered the most important for erection. A high frequency of normal findings on clinical examination of the lower limbs should thus be a guard against relying on such "routine neurological testing" to exclude a lesion to the cauda equina. Perianal sensation was, somewhat unexpectedly, also found to be poorly correlated with sexual function. This relatively poor correlation suggests that different populations of nerve fibres mediate perianal sensation and sexual function. Sensory loss in the lower sacral dermatomes is considered a common (perhaps even necessary) deficit in cauda equina lesions. Our study argues against sensory loss as an obligatory indicator of conus/cauda equina lesions, at least by the time of five or more months after the lesion. Three of 46 patients from our registers who definitely had a cauda equina lesion (as defined by history, clinical deficits, radiological data, and pathological denervation activity on a previous diagnostic EMG in their records) did not have any perianal sensory deficit on clinical examination. One of these patients had severely impaired sexual function, and the remaining two had slightly impaired function.

According to a recent consensus statement, ${ }^{21}$ concentric needle EMG and recording of the bulbocavernosus reflex are useful in selected patients with involvement of the sacral peripheral nervous system and urinary incontinence. It was one of our aims to evaluate the usefulness of these tests in predicting the presence of sexual dysfunction in patients with cauda equina lesions.
In our study, we assessed sacral reflex activity electromyographically. No clinical testing of the bulbocavernosus reflex was undertaken, as the EMG detection is more sensitive. ${ }^{22} \mathrm{We}$ elicited the sacral reflex response by touching the perianal skin bilaterally and detected it by a needle electrode inserted into the subcutaneous external anal sphincter muscle. ${ }^{7}$ It is probable that not only absent but also severely reduced sacral reflex activation on EMG corresponds to an absent bulbocavernosus reflex on clinical assessment. By touching the skin bilaterally, and inserting the needle bilaterally, this technique enabled us to test separately both (left/right) afferent and efferent pathways. ${ }^{7}$ This sacral reflex response correlated well with perianal sensory testing, but less well with sexual function.

To test the lower sacral segment motor fibres, we used quantitative EMG of the muscles supplied. Assessment of pathological spontaneous ("denervation") EMG activity and quantitative analysis of the interference pattern were not included in systematic evaluation of the external anal sphincter performed in our study, because the first was not expected to be informative in the chronic situation, and the second has been found to have only half the diagnostic sensitivity of MUP analysis techniques. ${ }^{10}$ The MUP count during relaxationwhich in this clinical situation provides a crude estimate of the number of motor units remaining after the neural lesion ${ }^{8}$ - did not predict the outcome of sexual function in our patients. Similarly, the analysis of MUP indices, which provide information on the external anal sphincter muscle reinnervation, was not correlated with sexual function. The reason for this finding may again lie in the different nerve fibre populations that are mainly responsible for each of these functions. They not only occupy a slightly different position within the cauda equina but they also show different resistance to compression (the parasympathetic fibres being thinner than the somatic fibres). Indeed in a recent study, testing for thin fibre function-such as testing thermal thresholds on the dorsal aspect of the penis-was reported to correlate better with erectile dysfunction in diabetic patients than nerve conduction studies testing thick nerve fibres. ${ }^{23}$

The common causes of the cauda equina damage (and those actually present in our study) are expected to produce an axonal type of nerve injury. Therefore uroneurophysiological tests of nerve conduction measuring latencies (bulbocavernosus reflex, pudendal nerve terminal latency test, pudendal somatosensory evoked potentials) are in fact not expected to be sensitive indicators of partial lesions, and thus to be even less well correlated with neurogenic sacral dysfunction. These tests were not performed in our study. On the basis of our 
results showing a poor correlation between EMG analysis and sexual dysfunction, we suggest it is highly improbable that any test for motor conduction would be a good indicator of sexual dysfunction. Similarly, because of the poor correlation of perianal sensory deficit and sexual dysfunction, we do not expect that tests measuring sensory latencies would be sensitive or specific for sexual dysfunction in cauda equina lesions. Because cauda equina lesions are located proximally to the dorsal root ganglia, amplitudes of the dorsal penile nerve sensory action potentials are expected to be normal. Although neurophysiological tests evaluating the autonomic nervous system would most directly test nerve fibres involved in sexual response, the penile sympathetic skin response does not correlate with erectile dysfunction in diabetic patients, ${ }^{23}$ and at this point we did not burden our patients with additional tests of unproven value.

Although neither perineal sensation nor MUP analysis was well correlated with sexual function (fig 3), these can support the diagnosis of a lesion to the cauda equina and provide important information about the severity, extent, and prognosis of the neural lesion.

Poor correlation of clinical and EMG abnormalities with sexual dysfunction might also reflect different abilities for repair of these nerve fibres after axonal lesions. This may particularly be the case with sensory fibres. Within the spinal canal these are anatomically the central axons leading from the body of the nerve cell to the spinal cord. In contrast to peripheral axons, central axons do not regenerate efficiently in spite of decompression. Several of our patients with severe perianal sensory loss and severe or complete denervation of the external anal sphincter on the early EMG showed efficient external anal sphincter reinnervation on follow up examination, without improvement in the perianal sensory loss.

It is remarkable that in our study only five of the 39 patients with sexual dysfunction (13\%) received medical attention specifically for this. Although we did not study this systematically, it seems that medical intervention could improve erectile function in this population, and oral sildenafil has previously been reported to be effective in individuals with spina bifida. ${ }^{24}$ However, treating erectile dysfunction did not increase the patients' satisfaction with sexual intercourse, possibly because medical intervention could not improve the genital sensory deficit. Nevertheless, a more active therapeutic approach to sexual dysfunction in patients with lesions to the cauda equina seems appropriate. Neurologists should play an important role as coordinators of comprehensive management of this neglected population of neurological patients, who need intervention and follow up by medical professionals in various different specialties.

\section{Conclusions}

Our study showed significant sexual dysfunction in men with lesions to the cauda equina. A detailed sexual history, clinical neurological examination, and EMG of the external anal sphincter muscle (MUP analysis) provided complementary information. We recommend performing all these diagnostic procedures in patients with an equivocal clinical picture. An abnormal finding in a particular procedure raises the possibility of another test being abnormal because of a neuropathic lesion, but the correlations are only moderate. Further studies are needed to shed more light on this somewhat neglected group of patients.

\section{ACKNOWLEDGEMENTS}

We thank Dr Aleš Pražnikar for help with recruitment of patients, Mićo Mrkaić PhD and Nacek Zidar for statistical analysis, and Dr Dianne Jones for language review. The study was supported by the Ministry of Education, Science and Sport of the Republic of Slovenia grant No J3 7899.

\section{Authors' affiliations}

S Podnar, D B Vodušek, Institute of Clinical Neurophysiology, Division of Neurology, University Medical Centre Liubliana, Slovenia

C Oblak, Department of Urology, Division of Surgery, University Medical Centre Ljubliana

Competing interests: none declared

\section{REFERENCES}

1 Larsen E, Hejgaard N. Sexual dysfunction after spinal cord or cauda equina lesions. Paraplegia 1984;22:66-74.

2 Hellstrom P, Kortelainen P, Kontturi M. Late urodynamic findings after surgery for cauda equina syndrome caused by a prolapsed lumbar intervertebral disk. J Urol 1986:135:308-12.

3 Shapiro S. Cauda equina syndrome secondary to lumbar disc herniation. Neurosurgery 1993;32:743-6.

4 Kennedy JG, Soffe KE, McGrath A, et al. Predictors of outcome in cauda equina syndrome. Eur Spine J 1999;8:317-22.

5 Nielsen B, de Nully M, Schmidt K, et al. A urodynamic study of cauda equina syndrome due to lumbar disc herniation. Urol Int 1980;35:167-70.

6 Rosen RC, Riley A, Wagner G, et al. An international index of erectile function (IIEF): a multidimensional scale for assessment of erectile dysfunction. Urology 1997:49:822-30.

7 Podnar S, Vodušek DB. Protocol for clinical neurophysiologic examination of pelvic floor. Neurourol Urodyn 2001;20:669-82.

8 Podnar S, Mrkaić M, Vodušek DB. Standardization of anal sphincter electromyography: quantification of continuous activity during relaxation. Neurourol Urodyn (in press).

9 Podnar S, Mrkaić M. Predictive power of motor unit potential parameters in anal sphincter electromyography. Muscle Nerve 2002;26:389-94.

10 Podnar S, Vodušek DB, Stålberg E. Standardization of anal sphincter electromyography: comparison of quantitative techniques of anal sphincter electromyography. Muscle Nerve 2002;25:83-92.

11 Stewart JD. Cauda equina disorders. In: Fowler CJ, Sakakibara R, Frohman EM, et al, eds. Neurologic bladder, bowel and sexual dysfunction. Amsterdam: Elsevier Science BV, 2001:63-74

12 Giuliano FA, Rampin O, Benoit G, et al. Neural control of penile erection. Urol Clin North Am 1995;22:747-66.

13 Rampin O, Bernabe J, Giuliano F. Spinal control of penile erection. World J Urol 1997;15:2-13

14 Brindley GS. Pathophysiology of erection and ejaculation. In: Whitfield $\mathrm{HN}$, Hendry WF, eds. Textbook of genito-urinary surgery. Edinburgh: Churchill Livingstone, 1985:1083-94.

15 Rushton DN. Sexual and sphincter dysfunction. In: Bradley WG, Daroff RB, Fenichel GM, et al, eds. Neurology in clinical practice. Boston: Butterworth-Heinemann, 1991:381-91.

16 Feldman HA, Goldstein I, Hatzichristou DG, et al. Impotence and its medical and psychosocial correlates: results of the Massachusetts male aging study. J Urol 1994;151:54-61.

17 Lavyne MH. Cauda equina syndrome secondary to lumbar disc herniation. Neurosurgery 1994;34:561.

18 Betts CD, Jones SJ, Fowler CJ. Erectile dysfunction in multiple sclerosis: associated neurological and neurophysiological deficits, and treatment of the condition. Brain 1994;117:1303-10.

19 Bors E, Comarr AE. Neurological disturbances of sexual function with special reference to 529 patients with spinal cord injuries. Urol Surv 1960;10:191-222.

20 Andersen BL, Broffitt B. Is there a reliable and valid self-report measure of sexual behavior? Arch Sex Behav 1988;17:509-25.

21 Vodušek DB, Bemelmans B, Chancellor $M$, et al. Clinical neurophysiology. In: Abrams P, Khoury S, Wein A, eds. Incontinence. Plymouth: Health Publication, 1999:155-95.

22 Blaivas JG, Zayed AA, Labib KB. The bulbocavernosus reflex in urology: a prospective study of 299 patients. J Urol 1981;126:197-9.

23 Lefaucheur JP, Yiou R, Colombel M, et al. Relationship between penile thermal sensory threshold measurement and electrophysiologic tests to assess neurogenic impotence. Urology 2001;57:306-9.

24 Palmer JS, Kaplan WE, Firlit CF. Erectile dysfunction in spina bifida is treatable. Lancet 1999;354:125-6. 